\title{
Hemorrhagic Brain Metastasis of Endometrial Stromal Sarcoma
}

\author{
Salih Gulsen ${ }^{{ }^{\star}}$, Aysen Terzi $^{2}$, Ozlem Isiksacan Ozen ${ }^{2}$, Ali Ayhan ${ }^{3}$ \\ ${ }^{1}$ Baskent University Medical Faculty Hospital - Neurosurgery, Maresal Fevzi Cakmak cad.10. sok. No: 45, Ankara 06540, \\ Turkey; ${ }^{2}$ Baskent University Medical Faculty - Pathology, Ankara, Turkey; ${ }^{3}$ Baskent University Medical Faculty - Obstetrics \\ and Gynecology, Ankara, Turkey
}

\begin{abstract}
Citation: Gulsen S, Terzi A, Ozen OI, Ayhan A. Citation: Gulsen S, Terzi A, Ozen Ol, Ayhan A Hemorrhagic Brain Metastasis of Endometrial
Stromal Sarcoma. OA Maced J Med Sci. 2014 $\begin{array}{ll}\text { Stromal Sarcoma. OA Maced J Med Sci. } 2014 \\ \text { Jun } & \text { 2(2):309-312. }\end{array}$ Jun
http://dx.doi.org/10.3889/oamjms.2014.052 Key words: stromal sarcoma; endometrium; cranium; metastasis; hemorrhage.

"Correspondence: Salih Gulsen, MD. Department of Neurosurgery, Faculty of Medicine, Baskent University Medical Faculty Department of Neurosurgery, Fevzi Cakmak Caddesi 10. Sokak no: 45, Cankaya Bahcelievler, Ankara 06490. Turkey. Business Phone: $00903122126868 / 1362$. FAX: 0090 3122237333. E.mail: salihgulsen07@gmail.com Received: 10-Feb-2014; Revised: 18-Mar2014; Accepted: 20-Mar-2014; Online first: 19-Apr-2014

Copyright: () 2014 Gulsen et al. This is an open access article distributed under the terms of the Creative Commons Attribution License, which permits unrestricted use, distribution, which pernits uniesticted use, distribution, original author and source are credited.

Competing Interests: The authors have declared that no competing interests exist.
\end{abstract}

\begin{abstract}
High-grade endometrial stromal sarcoma is a rare uterine malignancy. The cells of these tumors resemble endometrial cells in the proliferation stage, with many small plexiform arterioles. Herein we present a patient with endometrial stromal sarcoma that metastasized to the brain, along with the CT and MRI findings and a discussion of the surgical and histopathological features of the metastatic tumor. Immunohistopathological examination of the metastatic tumor showed a high mitotic rate and strong immunopositivity for CD10 and vimentin (as a mesenchymal marker); therefore, the lesion was considered to be high-grade endometrial stromal sarcoma. Due to the rarity of reported cases of metastatic brain tumors of high-grade endometrial stromal sarcoma, there isn't a standard treatment protocol; however, metastatic tumors of high-grade endometria stromal sarcoma match the same general concepts of brain metastasis. In addition, endometrial stromal sarcomas may be associated with hemorrhagic metastasis to the brain due to their pathologic characteristics.
\end{abstract}

\section{Introduction}

The brain is a common site of metastases; about 170,000 patients are newly diagnosed with brain metastases each year in the USA [1]. The occurrence of metastatic brain tumors is 10-fold greater than that of newly diagnosed primary brain malignancies [1-4]. Lung, breast, renal, and colorectal carcinoma, and malignant melanoma commonly metastasize to the brain [1-4]. Apart from choriocarcinoma, female genital tract cancers are a rare source of metastatic brain tumors [1-4]. Metastasis from the female genital tract to the brain is characteristic of widespread disseminated disease [5]. A PubMed search using 12 different key words in addition to the term endometrial stromal sarcoma showed that there were only 4 reported cases of endometrial stromal sarcoma that metastasized to the brain. Three of these cases were treated nonsurgically and one of them surgically, but none of them showed no hemorrhagic features at their metastasis in the brain. Herein we present what to the best of our knowledge is the first hemorrhagic cerebral metastasis of endometrial stromal sarcoma that was treated surgically.

\section{Case Report}

A 50-year-old female underwent abdominal hysterectomy with bilateral salpingo-oopherectomy in july 2012. Histopathological examination of the hysterectomy specimen showed endometrial stromal sarcoma. Microscopic examination of the hysterectomy specimen showed widespread invasion of the tumor into the vascular channels within the myometrium (Figures 1a, b). Adjuvant chemotherapy was initiated following abdominal hysterectomy. She 
presented to our hospital in April 262013 with abrupt onset of left-sided loss of power; she could not walk, or use her left arm and left hand. Her left arm was affected to a greater degree than her left leg. Her left leg power was 3/5, whereas her left arm power was 2/5. The patient's Glasgow Coma Scale score was $15 / 15$ at the time of presentation.

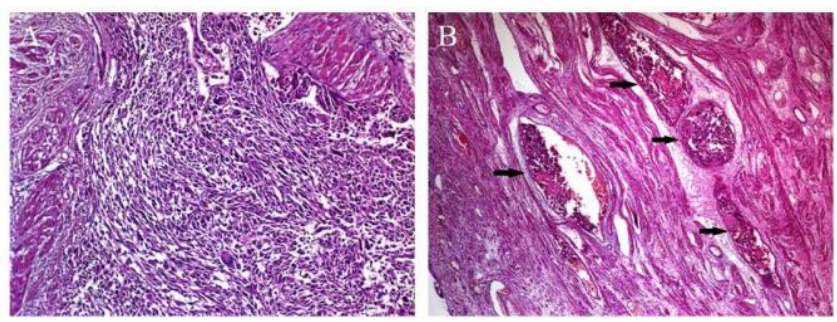

Figure 1: a) Hysterectomy specimen shows myometrial invasion by high-grade endometrial stromal sarcoma (H\&E, 100x). b) The black arrows indicate widespread tumoral invasion of the vascular channels within the myometrium (H\&E, 40x).

Cranial computed tomography (CCT) showed a lesion located in the right frontoparietal region (including the motor cortex) that appeared hyperdense with a perilesional hypodense area (Figure $2 \mathrm{a}, \mathrm{b}$ ). We then performed cranial magnetic resonance imaging (MRI), which showed heterogeneous contrast enhancement of the lesion in the right frontoparietal region. T1-weighted $\mathrm{MRI}$ showed that the legion extended into the right motor cortex at the right precentral gyrus. In addition, T2-weighted MRI showed perilesional edema (Figure 3a, b, c). Following the diagnosis of intracranial lesion, abdominal and thoracic computed tomography was performed and the findings were negative for metastasis. The patient's routine laboratory test results were normal.

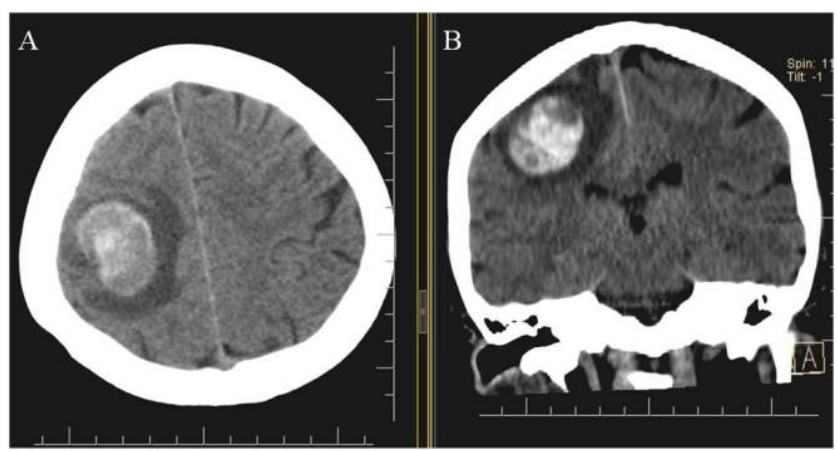

Figure 2: Axial (a), and coronal (b) non-contrast-enhanced CT shows a hematoma in the right frontoparietal lobe surrounded by hypodense edema.

The patient underwent frontoparietal craniotomy, with gross total extirpation of the tumor located in the right frontoparietal region in April 27 2013. The paracentral intersulcal region was incised at the parietal lobe and the tumor was located in the cortex at a depth of $5 \mathrm{~mm}$. Firstly, the hemorrhagic part of the tumor was aspirated, and then the wall of the tumoral cavity (all the way to the glial tissue) was aspirated. The tumoral tissue was hemorrhagic and infiltrated the glial tissue, with no obvious border between the tumor and glial tissue. It is noteworthy that the tumor was hemorrhagic, but that there wasn't any unusual bleeding during tumor removal. Lastly, bleeding in the surgical region was controlled and the cranium was closed in the typical manner. The patient was awakened and her left leg motor function was the same as pre surgery, but her left arm motor function was weaker than during the preoperative period - it was virtually plegic. She began to move the proximal part of her left arm and forearm postoperative second day, but could not move her left hand or fingers. We performed cranial MRI $24 \mathrm{~h}$ post-surgery; contrastenhanced T1-weighted MRI showed that there was no residual lesion. T2-weighted $\mathrm{MRI}$ showed that there was no edema (Figure $3 \mathrm{~d}, \mathrm{e}, \mathrm{f}$ ).
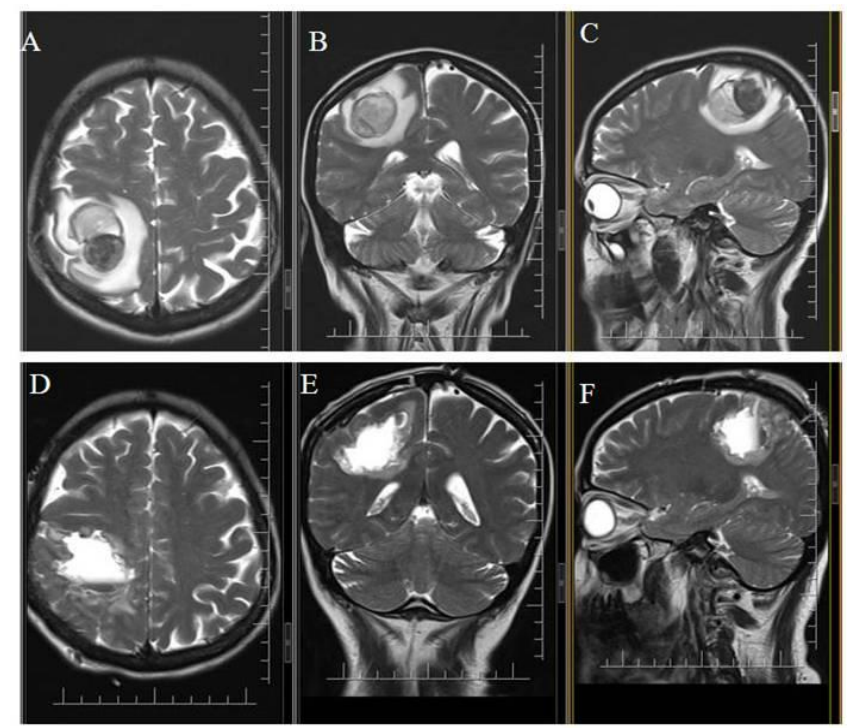

Figure 3: $a, b, c:$ T2-weighted MRI; the lesion measured $34 \times 39 \times$ $36 \mathrm{~mm}$ in 3 different sections. The lesion shows heterogeneous intensity surrounded by hyperintense edema, and it was located in the right frontoparietal lobe. In addition, it occupied the hand region of the right motor cortex; $d$, e, $f$ : T2-weighted MRI shows no edema, but there is a hyperintense appearance in area from which the metastatic brain tumor was removed.

Histopathological examination findings of the brain lesion specimen were consistent with endometrial stromal sarcoma (Figures 4a, b). Gross examination of the excised specimen showed a $2 \times 4$ $\mathrm{cm}$ yellow/cream-colored fragmented fragile mass with areas of necrosis and hemorrhage. Microscopic evaluation showed a metastatic malign mesenchymal neoplasm that had infiltrative margins, with several invasion foci into the adjacent glial tissue exhibiting marked reactive gliosis (Figure $4 a, b$ ). The neoplasm was composed of short bundles of ovoid to spindly cells, with marked nuclear anaplasia and scattered tumor giant cells (Figure $4 b, c)$. There was remarkable arborizing and pericytomatous vasculature, widespread necrosis and hemorrhagic areas, and high mitotic activity (Figure 4c). The metastatic brain 
neoplasm was strongly immunopositive for CD10 and vimentin, but was negative for CD34, smooth muscle actin, and cytokeratin AE1/AE3 (Figure 4d). The patient was scheduled to undergo radiotherapy and chemotherapy, but these adjuvant therapies are beyond of the scope for this case report.
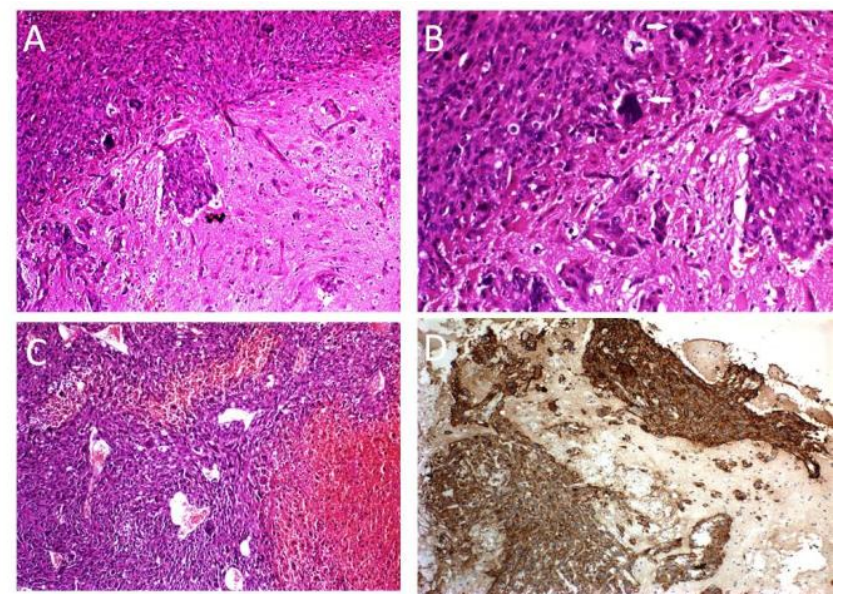

Figure 4: (a) Metastatic undifferentiated endometrial stromal sarcoma in the brain. Infiltrative neoplastic cell groups in the adjacent glial tissue (lower right side) show marked reactive gliosis (H\&E, 100x); (b) a close-up view of neoplastic ovoid to spindly cells, with anaplasia and neoplastic giant cells (white arrows) in the infiltrative tumor margin and reactive gliosis (H\&E, 400x); (c) the metastatic brain tumor exhibits a hemangiopericytomatous vascular pattern, with widespread necrosis and hemorrhage (H\&E, 100x); (d) the metastatic mesenchymal neoplasm in the brain was strongly immunopositive for CD10 (200x).

\section{Discussion}

Metastasis of female genital tract cancers occurs less frequently than in cases of other tumors, including lung, breast, renal, and colorectal carcinoma, and malignant melanoma. Endometrial stromal sarcoma is a rare tumor of the female genital tract; there are only approximately 450 new cases annually in the USA [1, 6]. Endometrial stromal sarcomas are histologically classified as low-grade or high-grade on the basis of the rate of mitosis [7-9]. Patients with low-grade endometrial stromal sarcoma have a 5 -year survival rate of $65 \%$, versus $<25 \%$ in those with high-grade disease $[10,11]$. Brain metastasis of endometrial stromal sarcoma is also rare because of the rarity of the tumor $[1,6]$. Genital tract cancers metastasize to the brain primarily via the hematogenous route [12]. Tumor cells that separate from the genital tract travel to the brain via the blood stream, through the inferior vena cava, right atrium, right ventricle, pulmonary artery, lungs, pulmonary veins, left atrium, left ventricle, and aorta [12, 13]. In addition, the Batson plexus (paravertebral venous plexus) is another route of dissemination of genital tract tumors [12, 13].

Microscopic examination of the presented patient's hysterectomy specimen showed that there was widespread invasion into the vascular channels within the myometrium (Figures 1a, b), which is evidence of hematogenous spread of the endometrial stromal sarcoma; however, the metastatic lesion's features was similar to that of a subacute intracerebral hematoma, as it was observed to be surrounded by edema via cranial CT. We think that the hemorrhagic appearance was due to the histopathological features that are characteristic of endometrial stromal sarcomas, as these tumors' cells resemble endometrial cells in the proliferation stage, with many small plexiform arterioles [2]. In addition, histopathology of the presented case showed arborizing and pericytomatous vasculature, which has been reported earlier (Figure 4a, b, c).

Cranial metastasis of endometrial stromal sarcoma has been reported 4 times by different authors until now. One case had metastasis in the sphenoid bone and lumbar spine [14], another case had lung and cerebral metastasis [15], and the third case had multiple cerebral metastases [16]. The last case has been recently reported in 2013, and the patient in this case had a lesion mimicking meningioma and located at right occipital lobe [17]. This lesion was successfully extracted from the occipital lobe [17]. Our case is $5^{\text {th }}$ case presented in the literature, and it is second case underwent surgery. However our presented case is the first reported case of hemorrhagic brain metastasis of endometrial stromal sarcoma underwent surgery. Histopathological examination of the specimen taken from the cerebrum also showed that the metastatic lesion was endometrial stromal sarcoma; however, extrauterine high-grade endometrial stromal sarcoma can be diagnostically challenging. In the presented case the pathologists performed immunohistochemical analysis in order to differentiate the endometrial stromal sarcoma from several soft tissue neoplasms with a hemangiopericytomatous vascular pattern. CD10 is a sensitive and diagnostically useful marker of endometrial stromal tumors [18]. The uterine tumor and metastatic tumor cells in the presented case exhibited strong immunopositivity for CD10 and vimentin (as a mesenchymal marker) [18]; this immune profile supported the diagnosis of endometrial stromal sarcoma. All of the presented patient's histopathological findings were consistent with endometrial stromal sarcoma.

In conclusion, in patients with endometrial stromal sarcoma cranial CT may show the presence of hemorrhagic metastasis and perilesional edema. In such cases contrast-enhanced MRI must be performed to differentiate between a hemorrhagic mass lesion and a hematoma of the cerebrum. Then, whether or not surgical treatment is necessary can be determined on the basis of the same general concepts of brain metastasis.

\section{References}

1. American Cancer Society. Cancer Facts and Figures 2009. Atlanta: American Cancer Society; 2009. Available from: 
http://www.cancer.org/ downloads/STT/500809web.pdf.

2. Madison T, Schottenfeld D, Baker V. Cancer of the corpus uteri in white and black women in Michigan, 1985-1994: an analysis of trends in incidence and mortality and their relation to histologic subtype and stage. Cancer. 1998;83:1546-54.

3. Sperduto PW, Berkey B, Gaspar LE, Mehta M, Curran W. A new prognostic index and comparison to three other indices for patients with brain metastases: an analysis of 1960 patients in the RTOG database. International Journal of Radiation Oncology Biology Physics. 2008; 70: 510- 4.

4. Tosoni A., Ermani M., Brandes A., The pathogenesis and treatment of brain metastases: a comprehensive review. Critical Reviews in Oncology/Hematology. 2004; 52: 199-215.

5. Acharya S, Hensley ML, Montag AC, Flaming GF. Rare uterine cancers. Lancet Oncol. 2005; 6:961-71.

6. Chan JK, Kawar NM, Shin JY, Osann K, Chen LM, Powell CB, et al. Endometrial stromal sarcoma: a population-based analysis. $\mathrm{Br} \mathrm{J}$ Cancer. 2008; 99:1210-8.

7. Haberal A, Kayıkcıoglu F, Boran N, Calıskan E, Ozgul N, Kose MF. Endometrial stromal sarcoma of the uterus: analysis of 25 patients. Eur J Obstet Gynecol Reprod Biol. 2003;109(2):209-13.

8. Inayama $Y$, Shoji A, Odagiri S, Hirahara F, Ito T, Kawano N, et al Detection of pulmonary metastasis of low-grade endometrial stromal sarcoma 25 years after hysterectomy. Pathol Res Pract. 2000;196:129-34

9. Amant F, Moerman P, Vergote I. The classification of a uterine sarcoma as 'High-grade endometrial stromal sarcoma' should be abandoned. Gynecol Oncol. 2004; 95:412-3.

10. Thomas MB, Keeney GL, Podratz KC, Dowdy SC. Endometrial stromal sarcoma: treatment and patterns of recurrence. Int $\mathrm{J}$ Gynecol Cancer. 2009; 19:253-6.

11. Nordal RR, Kristensen GB, Kaern J, et al. The prognostic significance of surgery, tumor size, malignancy grade, menopausa status, and DNA ploidy in endometrial stromal sarcoma. Gynecol Oncol. 1996;62:254-9.

12. Blecharz P, Urbański K, Mucha-Małecka A, Małecki K, Reinfuss $M$, Jakubowicz J, Skotnicki P. Hematogenous metastases in patients with Stage I or II endometrial carcinoma. Strahlenther Onkol. 2011;187(12):806-11,2011.

13. Ogawa $\mathrm{K}$, Yoshii $\mathrm{Y}$, Aoki $\mathrm{Y}$ et al. Treatment and prognosis of brain metastases from gynecological cancers. Neurologia MedicoChirurgica. 2008; 48: 57-62.

14. Huang MI, Debernardo RL, Rodgers M, Hart DJ. Endometrial stromal sarcoma metastasis to the lumbar spine and sphenoid bone. Rare Tumors. 2011;3(3):e27.

15. Akhavan A, Shishebor F, Moghimi M, Binesh F. Endometrial stromal sarcoma of uterus with metastasis to the lung and brain BMJ Case Rep. 2012;2012.

16. Merinsky A, Schmidt M, Heidner K, Koelbl H. Cerebral metastases of an endometrial stromal sarcoma: report of the first case. Onkologie. 2012; 35(5):272-4.

17. Stofko DL. Undifferentiated uterine sarcoma metastatic to the brain. Surg Neurol Int. 2013;27:4-12.

18. Bhargava R, Shia J, Hummer AJ, Thaler HT, Tornos C, Soslow RA. Distinction of endometrial stromal sarcomas from 'hemangiopericytomatous' tumors using a panel of immunohistochemical stains. Mod Pathol. 2005;18:40-7. 